\title{
EVOLUÇÃO SóCIO-PROFISSIONAL DE 140 PACIENTES EPILÉPTICOS SUBMETIDOS A TRATAMENTO MEDICAMENTOSO
}

\author{
Luís Marques-Assis *
}

Nenhuma doença crônica acarreta tantas implicações sociais e profissionais como a epilepsia. Tal fato é assinalado em todos os países do mundo, independentemente de seu grau de desenvolvimento. No Brasil o problema adquire importância maior, especialmente quando se recorda o fato de que numerosos pacientes, em virtude da moléstia, são obrigados a afastar-se do trabalho, tornando-se dependentes da família ou de Institutos de Aposentadoria, disso resultando graves prejuizos para a nação.

Por outro lado, pesquisas desenvolvidas em paises tècnicamente mais adiantados demonstram as dificuldades encontradas em centros especializados na recuperação de epilépticos ${ }^{6}$ e ressaltam o papel desempenhado pela severidade da epilepsia, em primeiro lugar, e pela constituição psicológica e nivel mental dos pacientes, em segundo lugar, no seu desempenho social e profissional $2,3,4, \overline{5}, \tau, 8,9$.

Neste trabalho são estudados, quanto à sua evolução sócio-profissional, pacientes epilépticos submetidos apenas a tratamento medicamentoso.

\section{MATERIAL, MÉTODO E RESULTADOS}

Foram estudados 140 pacientes acompanhados em Ambulatório de Epilepsia, sendo 74 do sexo feminino e 66 do sexo masculino, com idades entre 10 e 62 anos, sendo que $70 \%$ dos casos tinham idade compreendida entre 11 e 30 anos; 117 pacientes eram brancos, 16 eram pardos e 7 eram pretos. $O$ tempo de doença variou entre um e 34 anos. A freqüencia das crises oscilou entre mais de uma por dia e uma por mês. Apenas 7 casos apresentaram crises epilépticas não convulsivas; dos 133 casos restantes, em 25 a convulsão era pura e, em 108, precedida por manifestações focais; em 25 casos havia, além das convulsões, crises psicomotoras. Exame do liquido cefalorraqueano foi feito em 134 casos, tendo sido normal em todos. Eletrencefalograma, feito em todos os casos, foi normal em 24, apresentou anormalidades difusas em 33 e anormalidades focais em 83 casos.

Os pacientes foram tratados com medicamentos de fácil aquísição em nosso meio (barbitúricos, hidantoinatos, primidona e trimetadiona), isolada ou combinadamente. Em alguns casos foram associados tranquilizantes (clorpromazina, benzodiazepinicos ou butírofenonas). As doses dos antiepilépticos variaram de um a 10 comprimidos por dia; nos casos com doses elevadas era feita sempre associação de dois ou mais medicamentos. O tempo mínimo de tratamento foi de três anos.

Da Clinica Neurológica da Faculdade de Medicina da Universidade de São Paulo (Prof. Adherbal Tolosa), apresentado no III Congresso Brasileiro de Neurologia (Recife, 1968): * Médico assistente. 
Foram excluídos do estudo os casos com freqüencia de crises inferior a uma por mês. Sòmente foram incluídos os casos que apresentavam problemas sócio-profissionais por ocasião da primeira consulta e que responderam bem, do ponto de vista clinico, ao tratamento medicamentoso. Afora êste tratamento, foi feita apenas psicoterapia superficial de apoio.

Para o estudo foram consideradas como atividades básicas, a escolar, no que se refere às crianças de ambos os sexos, a doméstica, quanto aos pacientes de sexo feminino em idade adulta e a profissional, no que diz respeito aos pacientes adultos de sexo masculino. A evolução foi considerada favorável nos casos que se beneficiaram, do ponto de vista sócio-profissional, com a redução ou com a supressāo das crises epilépticas (melhora do rendimento escolar, melhor desempenho nas atividades do lar, iniciação ou maior rendimento nas atividades profissionais). Foram considerados reabilitados os casos que, apenas com a boa resposta clínica à terapêutica medicamentosa instituída, passaram a desenvolver ritmo de vida comparável ao dos individuos normais, nos diversos setores de suas atividades, alcançando posições e remunerações variáveis de acôrdo com suas aptidões.

A evolução sócio-profissional foi estudada em relação às manifestações clnicas, ao tempo de doença, à freqüência das crises e ao padrão eletrencefalográfico.

Os resultados, expressos em indices percentuais, podem ser verificados nos quadros 1 a 7 .

\section{COMENTARIOS}

Nos países mais evoluídos são considerados passíveis de reabilitação os doentes que responderam favoràvelmente, do ponto de vista clínico, ao tratamento medicamentoso bem planejado e bem executado. Mesmo assim, os resultados obtidos em Centros de Reabilitação devidamente aparelhados, recebendo pacientes rigorosamente estudados e selecionados, têm sido desapontadores ${ }^{6}$. No entanto, o problema não pode ser analisado de maneira semelhante no Brasil pois, em nosso meio, existe acentuado déficit no setor médico-assistencial ,o que propicia a manutenção da freqüência das crises em niveis elevados; tal fato, somado à maneira de encarar a doença por parte das famílias e da sociedade, de modo geral, e dos professôres e empregadores, em particular, favorece a instalação, a manutenção e a consolidação dos problemas sociais e profissionais que caracterizam a doença.

Como nos encontramos em estágio de desenvolvimento técnico-científico que não nos permite ainda aparelharmo-nos material e funcionalmente para promover adequadamente e de maneira mais direta a reabilitação de um grupo minoritário de pacientes epilépticos, suscetíveis de receber tal orientação, propusemo-nos, no presente trabalho, a atuar sôbre a severidade das epilepsias, fator incapcitante dos mais importantes, no sentido de avaliar os benefícios que adviriam do ponto de vista social e profissional. Para alcançar êsse objetivo, submetemos os pacientes estudados sòmente a tratamento medicamentoso; paralelamente, foi desenvolvida apenas psicoterapia superficial de apoio, que sempre deve ser feita, nessas casos.

A análise do quadro 1 demonstra que, de maneira geral, as formas convulsivas das epilepsia evoluíram melhor que as não convulsivas. Por outro lado (quadro 2) não houve diferenças nítidas da evolução nos pacientes com crises convulsivas puras em relação àquêles com aura. Quanto ao tempo de doença (quadro 3 ) deve-se ressaltar, inicialmente, a evolução menos favorável dos casos com menor tempo de doença, especialmente no 


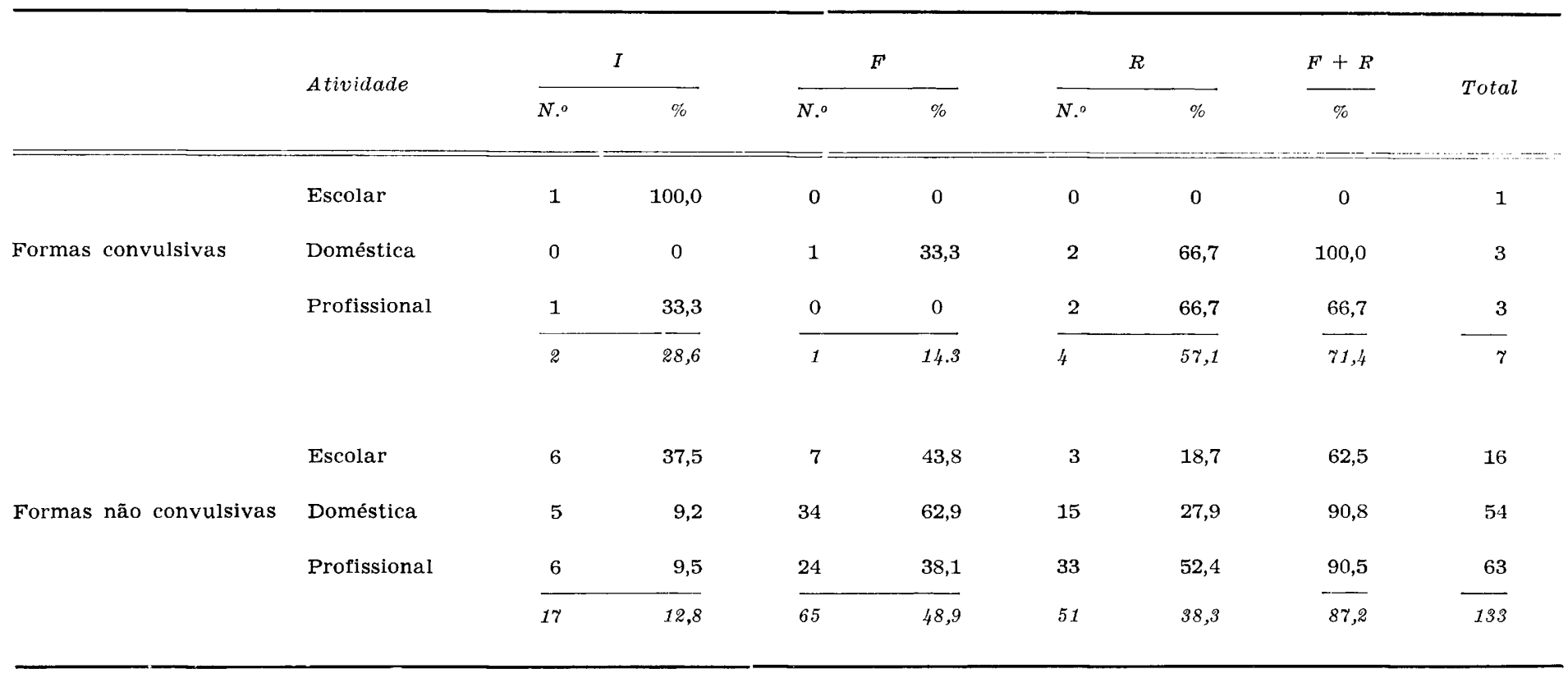

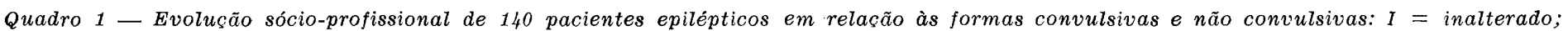

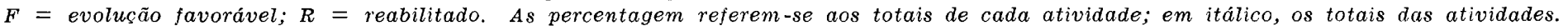




\begin{tabular}{|c|c|c|c|c|c|c|c|c|c|}
\hline & \multirow{2}{*}{ Atividade } & \multicolumn{2}{|c|}{$I$} & \multicolumn{2}{|c|}{$F$} & \multicolumn{2}{|c|}{$R$} & \multirow{2}{*}{$\frac{F+R}{\%}$} & \multirow{2}{*}{ Tota } \\
\hline & & $N .{ }^{\circ}$ & $\%$ & $N .^{\prime \prime}$ & $\%$ & $N .^{n}$ & $\%$ & & \\
\hline \multirow{4}{*}{ GM pura } & Escolar & 0 & 0 & 3 & 100,0 & 0 & 0 & 100,0 & 3 \\
\hline & Doméstica & 1 & 14,4 & 3 & 42,8 & 3 & 42,8 & 85,6 & 7 \\
\hline & \multirow[t]{2}{*}{ Profissional } & 2 & 13,3 & 4 & 26,7 & 0 & 60,0 & 86,7 & 15 \\
\hline & & 3 & 12,0 & 10 & 40,0 & 12 & 48,0 & 88,0 & 25 \\
\hline \multirow{4}{*}{ GM com aura } & Escolar & 5 & 41.7 & 4 & 33,3 & 3 & 25,0 & 58,3 & 12 \\
\hline & Doméstica & 2 & 4,4 & 31 & 68,9 & 12 & 27,6 & 95,6 & 45 \\
\hline & \multirow[t]{2}{*}{ Profissional } & 7 & 13,7 & 20 & 39,2 & 24 & 47,1 & 86,3 & 51 \\
\hline & & 14 & 13,0 & 55 & 50,9 & 39 & 36,1 & 87,0 & 108 \\
\hline
\end{tabular}

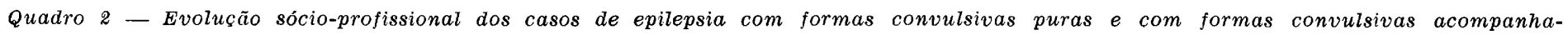
das de aura: $I=$ inalterado; $F=$ evolução favorável $R=$ reabilitado. 


\begin{tabular}{|c|c|c|c|c|c|c|c|c|c|}
\hline & \multirow{2}{*}{ Atividade } & \multicolumn{2}{|c|}{$I$} & \multicolumn{2}{|c|}{$F$} & \multicolumn{2}{|c|}{$R$} & \multirow{2}{*}{$\frac{F+R}{\%}$} & \multirow{2}{*}{ Total } \\
\hline & & $N .{ }^{o}$ & $\%$ & $N .{ }^{\circ}$ & $\%$ & $N .^{\circ}$ & $\%$ & & \\
\hline & Escolar & 4 & 40,0 & 6 & 60,0 & 0 & 0 & 60,0 & 10 \\
\hline \multirow[t]{4}{*}{ Até 3} & Doméstica & 0 & 0 & 2 & 33,3 & 4 & 66,7 & 100,0 & 6 \\
\hline & Profissional & 3 & 33,3 & 3 & 33,3 & 3 & 33,3 & 66,6 & 9 \\
\hline & & 7 & 28,0 & 11 & 44,0 & 7 & 28,0 & 72,0 & 25 \\
\hline & Escolar & 2 & 50,0 & 1 & 25,0 & 1 & 25,0 & 50,0 & 4 \\
\hline \multirow[t]{4}{*}{ De 3 a 10} & Doméstica & 1 & 5,3 & 13 & 68,4 & 5 & 26,3 & 94,7 & 19 \\
\hline & Profissional & 1 & 4,5 & 10 & 45,5 & 11 & 50,0 & 95,5 & 22 \\
\hline & & 4 & 8,8 & 24 & 53,3 & 17 & 37,9 & 91,2 & 45 \\
\hline & Escolar & 0 & 0 & 0 & 0 & 2 & 100,0 & 100,0 & 2 \\
\hline \multirow[t]{3}{*}{ Mais que 10} & Doméstica & 2 & 6,7 & 20 & 66,6 & 8 & 26,7 & 93,3 & 30 \\
\hline & Profissional & 6 & 15,8 & 11 & 28,9 & 21 & 55,3 & 84,2 & 38 \\
\hline & & 8 & 11,4 & 31 & 44,3 & 31 & 44,3 & 88,6 & 70 \\
\hline
\end{tabular}

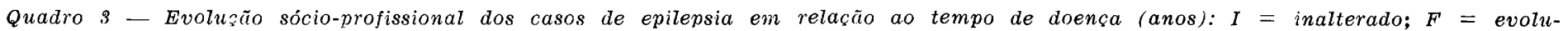
ção favorável; $R=$ reabilitado. 


\begin{tabular}{|c|c|c|c|c|c|c|c|c|c|}
\hline & \multirow{2}{*}{ Atividade } & \multicolumn{2}{|c|}{$I$} & \multicolumn{2}{|c|}{$F$} & \multicolumn{2}{|c|}{$R$} & \multirow{2}{*}{$\frac{F+R}{\%}$} & \multirow{2}{*}{ Total } \\
\hline & & $N .^{o}$ & $\%$ & $N .^{\circ}$ & $\%$ & $N .^{\circ}$ & $\%$ & & \\
\hline \multirow{4}{*}{ Até $7 / 7$} & Escolar & 4 & 80,0 & 1 & 20,0 & 0 & 0 & 20,0 & 5 \\
\hline & Doméstica & 0 & 0 & 18 & 69,2 & 8 & 30,8 & 100,0 & 26 \\
\hline & Profissional & 6 & 18,2 & 10 & 30,3 & 17 & 51,5 & 81,8 & 33 \\
\hline & & 10 & 15,5 & 29 & 45,3 & 25 & 39,1 & 84,4 & 64 \\
\hline \multirow{4}{*}{$8 / 8$ a $15 / 15$} & Escolar & 1 & 14,3 & 4 & 57,1 & 2 & 28,6 & 85,7 & 7 \\
\hline & Doméstica & 0 & 0 & 5 & 41,7 & 7 & 58,3 & 100,0 & 12 \\
\hline & Profissional & 1 & 4,5 & 12 & 54,5 & 9 & 41,0 & 95,5 & 22 \\
\hline & & 2 & 4,9 & 21 & 51,2 & 18 & 43,9 & 95,1 & 41 \\
\hline \multirow{4}{*}{$16 / 16$ a $30 / 30$} & Escolar & 1 & 25,0 & 2 & 50,0 & 1 & 25,0 & 75,0 & 4 \\
\hline & Doméstica & 3 & 17,6 & 12 & 70,6 & 2 & 11,8 & 82,4 & 17 \\
\hline & Profissional & 3 & 21,4 & 2 & 14,3 & 9 & 64,3 & 78,6 & 14 \\
\hline & & 7 & 20,0 & 16 & 45,7 & 12 & 34,3 & 80,0 & 35 \\
\hline
\end{tabular}

Quadro 4 - Evolução sócio-profissional dos casos de epilepsia em relação à freqüência (dias) das crises: $I=$ inalterado; $F$ = evolução favorável; $R=$ reabilitado. 


\begin{tabular}{|c|c|c|c|c|c|c|c|c|c|}
\hline \multirow{2}{*}{$E E G$} & \multirow{2}{*}{ Atividade } & \multicolumn{2}{|c|}{$I$} & \multicolumn{2}{|c|}{$F$} & \multicolumn{2}{|c|}{$R$} & \multirow{2}{*}{$\frac{F+R}{\%}$} & \multirow{2}{*}{ Total } \\
\hline & & $N .^{\circ}$ & $\%$ & $N .{ }^{\circ}$ & $\%$ & $N .^{o}$ & $\%$ & & \\
\hline \multirow{4}{*}{ Normal } & Escolar & 2 & 100,0 & 0 & 0 & 0 & 0 & 0 & 2 \\
\hline & Doméstica & 1 & 7,7 & 8 & 61,5 & 4 & 30,8 & 92,3 & 13 \\
\hline & Profissional & 1 & 10,0 & 5 & 50,0 & 4 & 40,0 & 90,0 & 10 \\
\hline & & 4 & 16,0 & 13 & 52,0 & 8 & 32,0 & 84,0 & 25 \\
\hline \multirow{4}{*}{ Focal } & Escolar & 4 & 33,3 & 5 & 41,7 & 3 & 25,0 & 66,7 & 12 \\
\hline & Doméstica & 2 & 5,9 & 22 & 64,7 & 10 & 29,4 & 94,1 & 34 \\
\hline & Profissional & 6 & 16,2 & 14 & 37,8 & 17 & 46,0 & 83,8 & 37 \\
\hline & & 12 & 14,5 & 41 & 49,4 & 30 & 36,1 & 85,5 & 83 \\
\hline \multirow{4}{*}{ Difuso } & Escolar & 0 & 0 & 2 & 100,0 & 0 & 0 & 100,0 & 2 \\
\hline & Doméstica & 0 & 0 & 5 & 62,5 & 3 & 37,5 & 100,0 & 8 \\
\hline & Profissional & 3 & 13,6 & 5 & 22,7 & 14 & 63,7 & 86,4 & 22 \\
\hline & & 3 & 9,4 & 12 & 37,5 & 17 & 53,1 & 90,6 & 32 \\
\hline
\end{tabular}

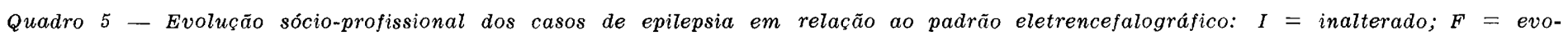
lução favorável; $R=$ reabilitado. 


\begin{tabular}{|c|c|c|c|c|c|c|c|c|c|}
\hline & \multirow{2}{*}{ Atividade } & \multicolumn{2}{|c|}{$I$} & \multicolumn{2}{|c|}{$F$} & \multicolumn{2}{|c|}{$R$} & \multirow{2}{*}{$\frac{F+R}{\%}$} & \multirow{2}{*}{ Total } \\
\hline & & $N .{ }^{\circ}$ & $\%$ & $N .{ }^{\circ}$ & $\%$ & $N .^{\circ}$ & $\%$ & & \\
\hline \multirow{4}{*}{ Temporal } & Escolar & 2 & 40,0 & 1 & 20,0 & 2 & 40,0 & 60,0 & 5 \\
\hline & Doméstica & 1 & 3,6 & 18 & 64,3 & 9 & 32,1 & 96,4 & 28 \\
\hline & \multirow[t]{2}{*}{ Profissional } & 6 & 19,4 & 13 & 41,9 & 12 & 38,7 & 80,6 & 31 \\
\hline & & 9 & $1 /, 1$ & 32 & 50,0 & 23 & 35,9 & 85,9 & 64 \\
\hline \multirow{4}{*}{ Temporal esquerdo } & Escolar & 2 & 50,0 & 0 & 0 & 2 & 50,0 & 50,0 & 4 \\
\hline & Doméstica & 0 & 0 & 4 & 36,4 & 7 & 63,6 & 100,0 & 11 \\
\hline & \multirow[t]{2}{*}{ Profissional } & 4 & 26,7 & 4 & 26,7 & 7 & 46,6 & 73,3 & 15 \\
\hline & & 6 & 20,0 & 8 & 26,7 & 16 & 53,3 & 80,0 & 30 \\
\hline
\end{tabular}

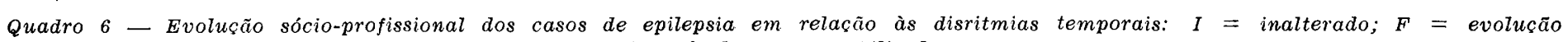
favorável; $R=$ reabilitado.

\begin{tabular}{|c|c|c|c|c|c|c|c|c|}
\hline \multirow{2}{*}{ Atividade } & \multicolumn{2}{|c|}{$I$} & \multicolumn{2}{|c|}{$F$} & \multicolumn{2}{|c|}{$R$} & \multirow{2}{*}{$\frac{F+R}{\%}$} & \multirow{2}{*}{ Total } \\
\hline & $N .^{\circ}$ & $\%$ & $N .{ }^{\circ}$ & $\%$ & $N .{ }^{\circ}$ & $\%$ & & \\
\hline Escolar & 6 & 37,5 & 7 & 43,7 & 3 & 18,8 & 62,5 & 16 \\
\hline Doméstica & 3 & 5,4 & 35 & 63,6 & 17 & 31,0 & 94,6 & 55 \\
\hline \multirow[t]{2}{*}{ Profissional } & 10 & 14,5 & 24 & 34,8 & 35 & 50,7 & 85,5 & 69 \\
\hline & 19 & 13,6 & 66 & 47,1 & 55 & 39,3 & 86,4 & 140 \\
\hline
\end{tabular}

Quadro 7 - Evolução sócio-profissional geral dos 140 casos de cpilepsia estudados. 
que se refere às atividades profissionais; por outro lado, os melhores resultados ocorreram no grupo de pacientes com tempo de doença compreendido entre 3 e 10 anos; não encontramos explicação para êsse fato, aparentemente paradoxal. Em relação à severidade da epilepsia, traduzida pela freqüência das crises (quadro 4), o menor índice percentual de casos reabilitados correspondeu às formas menos severas de epilpesia; os melhores resultados foram obtidos nas formas intermediárias. Em relação a êsses achados, é compreensivel que as formas clìnicamente mais benignas de epilepsia, que apresentavam problemas sócio-profissionais prévios, se beneficiassem menos, sob êste ponto de vista, com a eliminação ou com a redução das crises; é possivel que sejam os pacientes dêsse grupo que mereçam orientação especializada no sentido de se obter sua reabilitação. Em relação ao padrão eletrencefalográfico (quadro 5), foi verificada melhor evolução dos pacientes com disritmia difusa. Quanto ao pacientes com epilepsia temporal (quadro 6), a evolução foi, de modo geral, favorável; dêsse grupo, o estudo dos pacientes com foco temporal esquerdo (a forma mais freqüente das epilepsias temporais) mostrou que, embora tivesse havido maior índice percentual de casos reabilitados, a evolução não foi tão favorável. Finalmente, pela análise global dos casos estudados (quadro 7), pode-se verificar que os resultados mais precários foram obtidos em relação aos pacientes que desenvolviam atividades escolares e, os melhores, nos pacientes com atividades domésticas. De qualquer forma, os resultados favoráveis obtidos nas três atividades estudadas, permitem certo otimismo nas conclusões, sendo lícito supor que a maioria dos pacientes epilépticos, uma vez que sejam convenientemente tratados do ponto de vista clínico e superficialmente amparados do ponto de vista psicológico, está em condições de desenvolver vida em ritmo próximo do normal, não exigindo medidas especializadas para que sua reabilitação seja alcançada.

\section{RESUMO}

É estudada a evolução sócio-profissional de 140 doentes epilépticos, submetidos apenas a tratamento medicamentoso. Foram empregadas drogas de fácil aquisição em nosso meio (barbitúricos, hidantoinatos, primidona e trimetadiona), utilizadas isolada ou combinadamente. No estudo foram consideradas bàsicamente as atividades escolares, domésticas e profissionais. A evolução sócio-profissional foi estudada em relação às manifestações clínicas, ao tempo de doença, à freqüência das crises e ao padrão eletrencefalográfico. Os resultados, expressos em índices percentuais, permitiram ao autor concluir que na maioria dos pacientes epilépticos, convenientemente tratado 3 do ponto de vista clínico, os problemas sociais e profissionais podem ser corrigidos ou evitados, independentemente de outras medidas especializadas que possam ser postas em prática. 
The socio-professional evolution of 140 epileptic patients submitted to antiepileptic drug therapy.

The socio-professional evolution of 140 epileptic patients submitted to antiepileptic drug therapy is studied. Only barbiturates, hydantoin, primidone and trimethadione were administered to the patients, isolated or in association. The school, house keeper and professional activities were considered in the investigation. The socio-professional follow-up was investigated regarding to clinic manifestations, time of disease, frequency of seizures and electroencephalographic pattern. The results, analysed in percentage, led the author to the conclusion that in most epileptic patients, adequately controlled with drugs, the social and the professional problems can be avoided.

\section{REFERENCIAS}

1. AsSis, J. L. - Epilepsia e Previdência Social. Rev. Ass. med. bras. 9:391-400, 1963.

2. BIRKENHEAD, C. - Epilepsy as a social problem. Brit. med. J. 1:672-675, 1968.

3. DENNERLL, R. D.; RODIN, E. A.; GONZALES, S.; SCHWARTZ, M. L. \& LIN, Y. - Neurological and psychological factors related to employability of persons with epilepsy. Epilepsia 7:318-329, 1966.

4. GOODGLASS, H.; MORGAN, M.; FOLSOM, A. T. \& GUADFASEL, F. A. Epileptic seizures. Psychological factors and occupational adjustment. Epilepsia 4:322-341, 1963.

5. JUUL-JENSEN, P. - - Socio-medical problems of epileptics. A preliminary report. Epilepsia 2:197-206, 1961.

6. JUUL-JENSEN, P. - Vocational training of epileptics. Epilepsia 2:291-296, 1961.

7. LENNOX, W. C. \& LENNOX, M. A. - Epilepsy and Related Disorders. LittleBrow Co., Boston, 1960.

8. POND, D. A. \& BIDWELL, B. H. - A survey of epilepsy in fourteen general practices: social and psychological aspects. Epilepsia 1:285-299, 1959/60.

9. WADA, T. - Socio-medical aspects of epilepsy in Japan. Epilepsia 7:73-79, 1966.

Clinica Neurológica - Faculdade de Medicina da Universidade de Sĩo Paulo Caixa Postal 3161 - São Paulo, SP - Brasil. 